\section{Necessidade insatisfeita por métodos anticoncepcionais no Brasil}

\section{Unmet contraception needs in Brazil}

Luciane Santiago Tavares ${ }^{1}$

Iuri da Costa Leite ${ }^{2}$

Fernando Salgueiro Passo Telles ${ }^{2}$

${ }^{1}$ Curso de Saúde Pública, Escola Nacional de Saúde Pública (ENSP/FIOCRUZ)

Escola Nacional de Saúde Pública (ENSP/FIOCRUZ)

\section{Resumo}

No Brasil, a prevalência do uso de contraceptivo é elevada e a taxa de fecundidade total está quase no nível de reposição. No entanto, ainda há um percentual significativo de gravidezes indesejadas, abortos provocados e mulheres que não querem mais ter filhos ou querem tê-los mais tarde, porém não estão usando métodos contraceptivos - ou seja, mulheres com necessidade insatisfeita por anticoncepção. O objetivo deste estudo é apresentar a magnitude da necessidade insatisfeita por método anticoncepcional entre mulheres unidas no Brasil, assim como identificar os fatores a ela associados. A fonte de dados utilizada nesse estudo é proveniente da Pesquisa Nacional Sobre Demografia e Saúde, realizada no Brasil em 1996. Para identificar os determinantes associados com a necessidade insatisfeita por anticoncepção foi utilizado o modelo multinomial, em que a variável resposta tem três categorias: necessidade para espaçar, necessidade para limitar e sem necessidade. A análise empreendida ao longo deste trabalho revelou uma forte associação entre as variáveis relativas ao ciclo de vida das mulheres (idade e número de filhos) e a necessidade insatisfeita por métodos anticoncepcionais. Com relação às variáveis socioeconômicas destaca-se a elevada probabilidade de necessidade insatisfeita de limitar entre as mulheres da região Nordeste. Observou-se ainda que mulheres com maior nível de escolaridade são menos propensas a apresentar uma necessidade insatisfeita de limitar. A análise aponta para a necessidade de adequação e universalização dos serviços de planejamento familiar com vistas a um atendimento integral à saúde da mulher.

Palavras-chave: Necessidade insatisfeita por métodos anticoncepcionais. Planejamento familiar. Brasil.

Correspondência: Luciane Santiago Tavares. Rua Alice 194 / 703 Laranjeiras - Rio de Janeiro, RJ CEP: 22240-021. E-mail: Istavares@globo.com 
Abstract

In Brazil the prevalence of use of contraceptives is high and the total fertility rate is almost at the replacement level. However, there still is a significant proportion of unwanted pregnancies, induced abortions, and women who do not want any more children, or those who want to postpone childbearing, but are not using any conceptive method, that is, women with an unmet contraceptive need. The objective of this study is to describe the magnitude of the unmet contraceptive needs among Brazilian married women, as well as to identify the factors associated with this need. The information used is from the National Demographics and Health Survey carried out in Brazil in 1996. In order to identify the determinants associated with the unmet contraceptive needs, a multinomial model was used, with the outcome variable classified into three categories: need for spacing births, need for limiting births, and no need. The analysis conducted along with this work showed a strong association between factors related to the life cycle (age and number of children) and unmet contraception needs. In relation to socioeconomic factors, the high likelihood of unmet contraceptive needs among women from the Northeast region stands out. It has also been shown that women with a higher educational level are less prone to have an unmet need for limiting births. The analysis points out the need of an appropriate and universal program for family planning services to assure full support to women's health.

Keywords: Unmet contraceptive needs. Family Planning. Brazil.

\section{Introdução}

O declínio da fecundidade observado no Brasil deu-se de forma muito rápida, tendo a taxa de fecundidade total (TFT) passado de 6,3 filhos em 1960 para 2,2 filhos em $2001^{1}$, patamar bem próximo do nível de reposição ${ }^{1}$.

$\mathrm{O}$ uso de métodos contraceptivos é reconhecido como a variável intermediária de maior importância na determinação dos níveis de fecundidade ${ }^{2-4}$. $\mathrm{O}$ aborto induzido também cumpriu um papel relevante na queda da fecundidade ${ }^{2}$, porém sua ilegalidade no Brasil dificulta dimensionar a magnitude deste evento. Apesar de não haver estatísticas oficiais sobre as práticas abortivas, segundo o trabalho "Clandestine Abortion" do Instituto Alan Guttmacher, a taxa de aborto no Brasil foi estimada em 3,7 por 100 mulheres em idade fértil ${ }^{5}$.

A Pesquisa Nacional sobre Demografia e Saúde, 1996 (PNDS - 1996) aponta que $76,7 \%$ das mulheres unidas (mulheres que se declararam como vivendo numa união estável, seja formal ou informal) faziam uso de algum método contraceptivo, sendo $62,7 \%$ para limitar e $14,0 \%$ para espaçar os nascimentos ${ }^{6}$. Estas taxas de prevalência do uso de anticoncepcionais são similares àquelas dos países desenvolvidos. Contudo, observamos que o uso de contraceptivos concentra-se em dois métodos anticoncepcionais: a ligadura tubária e a pílula. A esterilização feminina representa $52,3 \%$ de todos os métodos contraceptivos utilizados entre as mulheres unidas, seguida pela pílula, cuja prevalência situa-se em torno de $27,0 \%{ }^{6}$. A esterilização masculina, por outro lado, é menos comumente praticada $(3,4 \%)$ do que métodos tradicionais como a abstinência periódica $(4,0 \%)$ e o coito interrompido $(4,0 \%)^{6}$.

Por outro lado, apesar da grande maioria das mulheres unidas utilizarem algum método contraceptivo, ainda há um percentual significativo de gravidezes não desejadas. Segundo esta mesma pesquisa, aproximadamente $50 \%$ dos nascimentos 
ocorridos nos últimos cinco anos anteriores à data da pesquisa não foram planeja$\operatorname{dos}^{6}$. Além disso, existe também um grupo de mulheres unidas, férteis e desejosas de espaçar ou limitar nascimentos que não estavam usando qualquer tipo de método anticoncepcional, bem como grávidas ou amenorréicas que não tinham planejado a sua atual ou última gravidez. Na literatura, essas mulheres têm sido classificadas como tendo uma necessidade insatisfeita por anticoncepção ${ }^{7}$.

A forte concentração em apenas dois métodos contraceptivos, pílula e esterilização, somada à discrepância existente entre a preferência e o comportamento reprodutivo pode ser atribuída, em grande parte, à ausência de um programa de planejamento familiar que sustente essa demanda total por métodos anticoncepcionais, isto é, das mulheres usuárias de métodos contraceptivos e daquelas com necessidade insatisfeita por anticoncepção ${ }^{8}$.

No entanto, no Brasil, o planejamento familiar tem amparo na constituição federal e é regulamentado pelas leis específicas referentes aos direitos reprodutivos.

O Ministério da Saúde criou em 1983 o Programa de Assistência Integral à Saúde da Mulher (PAISM), que indicava o Estado como provedor de meio e informação para que todos os brasileiros pudessem planejar suas famílias ${ }^{9}$. Em 1988, a Constituição Brasileira contemplou a saúde como direito do cidadão e o livre-arbítrio do planejamento familiar, competindo ao Estado a responsabilidade de prover recursos para o exercício desses direitos. O Sistema Único de Saúde (SUS) foi o instrumento criado para garantir a saúde da população ${ }^{9}$. No que diz respeito ao planejamento familiar, anos mais tarde foi criada a lei $\mathrm{n}^{\circ}$ $9.0263 / 96^{9}$, que regulamenta o direito constitucional e contempla um conjunto de ações para assistência à concepção e contracepção.

Apesar de toda a legislação existente no Brasil, o reduzido leque de métodos contraceptivos e a discrepância existente entre preferência e comportamento repro- dutivo acima descritos violam o princípio básico de autonomia individual e/ou familiar que valoriza a livre escolha.

Neste sentido, a Conferência Internacional sobre População e Desenvolvimento (CPID), realizada no Cairo em 1994, da qual o Brasil foi signatário, recomenda que todos os países devem identificar e remover as principais barreiras que ainda persistem para o uso efetivo dos serviços de planejamento familiar. Em 1999, na ocasião da revisão do $5^{\circ}$ ano da CPID, os governos estabeleceram uma meta de eliminação da necessidade insatisfeita por métodos contraceptivos até $2015^{10}$.

O objetivo deste estudo é apresentar a magnitude da necessidade insatisfeita por método anticoncepcional entre mulheres unidas para o Brasil, assim como identificar os fatores a ela associados utilizando informações da última pesquisa, com representatividade nacional, realizada em 1996. As estimativas da necessidade insatisfeita por métodos contraceptivos e o conhecimento de seus fatores condicionantes podem facilitar a tomada de decisões, ao estabelecer prioridades de investimentos em políticas públicas, visando atender as necessidades dessa parcela da população que está sob risco de uma gravidez indesejada.

\section{Material e métodos}

\section{Fonte de dados}

Os dados utilizados neste estudo são provenientes da Pesquisa Nacional Sobre Demografia e Saúde (PNDS). Esta foi realizada no Brasil, em 1996, pela Sociedade Civil Bem-Estar Familiar no Brasil (BEMFAM), em conjunto com o Instituto para o Desenvolvimento de Recursos (IRD), como parte do Programa "Demographic and Health Survey” (DHS) ${ }^{6}$. A coleta de dados da PNDS baseou-se em entrevistas domiciliares, com um sistema de questionários preenchidos por meio de entrevistas individuais de 12.612 mulheres entre 15 e 49 anos. Entre as mulheres entrevistadas, $60 \%$ eram casa- 
das ou viviam em união ${ }^{6}$ A pesquisa obteve informações sobre níveis de fecundidade, mortalidade infantil e materna, anticoncepção, saúde da mulher e da criança, conhecimento e atitudes relacionadas às doenças sexualmente transmissíveis e AIDS, além de dados que permitem uma caracterização sociodemográfica da população ${ }^{6}$.

\section{Conceito de necessidade insatisfeita}

A idéia de necessidade insatisfeita por métodos anticoncepcionais surgiu na década de sessenta, quando dados das pesquisas sobre o conhecimento, as atitudes, e as práticas relativas à contracepção (Knowledge, Attitudes, and Practices $K A P$ ) mostraram uma lacuna, para um contingente significativo de mulheres, entre intenções reprodutivas e comportamento contraceptivo $(K A P \text {-gap })^{11}$.

Essa noção ganhou importância conceitual durante os anos 70, tendo como base os dados das primeiras Pesquisas Mundiais de Fecundidade (World Fertlity Surveys - WFS), onde o foco de interesse concentrava-se na preferência da fecundidade e do uso de contraceptivo. Nas décadas subseqüentes, a abordagem do conceito "necessidade insatisfeita por métodos contraceptivos" recebeu diversas modificações, procurando-se levar em conta a exposição ao risco de engravidar, ou seja, classificando as mulheres em fecundas e infecundas ${ }^{12,13}$

Nas Pesquisas sobre Prevalência de Contracepção (Contraceptive Prevalence Survey - CPS), conduzidas entre os anos de 1970 e 1984, foram contempladas perguntas sobre o interesse das mulheres casadas em espaçar o nascimento dos filhos. Assim tornou-se possível calcular a necessidade insatisfeita por anticoncepção para espaçar e para limitar os nascimentos, possibilitando distinguir o interesse potencial em métodos provisórios daqueles permanentes ou de longo prazo, que são de maior interesse quando temos necessidade de limitar os nascimentos $^{14,15}$.
No Programa de Pesquisa Demografia e de Saúde - DHS (Demographic and Health Survey), conduzido na década de 80 nos países em desenvolvimento, houve um refinamento dos dados que possibilitou classificar as mulheres grávidas e amenorréicas em função de três critérios: se a gravidez foi planejada (necessidade satisfeita), se a gravidez não foi desejada naquele momento (necessidade para espaçar) e se a mulher não desejava ter outro filho (necessidade para limitar). Estabeleceu-se ainda que as mulheres cujas gravidezes foram decorrentes de falha no uso do método anticoncepcional não seriam captadas como tendo necessidade insatisfeita por anticoncepção, pois as informações da pesquisa não permitem distinguir entre falha do método e falha na forma como o método foi utilizado ${ }^{12}$.

O presente artigo utiliza o conceito de necessidade insatisfeita por métodos anticoncepcionais proposto por Westoff e Ochoa $^{7}$. Segundo os autores, considera-se necessidade insatisfeita de anticoncepção o conjunto de mulheres casadas ou unidas, não usuárias de métodos anticoncepcionais, férteis e desejosas de espaçar ou limitar os nascimentos, bem como as grávidas ou amenorréicas, que não tenham planejado a sua atual ou última gravidez e não estavam usando nenhum método anticoncepcional quando engravidaram.

\section{Modelo}

Do ponto de vista metodológico, para identificar os determinantes associados com a necessidade insatisfeita por anticoncepção usamos o modelo multinomial, pois a variável resposta possui três categorias nominais: necessidade para espaçar, necessidade para limitar e sem necessidade.

A PNDS apresenta uma estrutura hierárquica, na medida em que mulheres de uma mesma comunidade devem apresentar atitudes e comportamentos semelhantes, o que invalida o pressuposto de independência entre as observações ${ }^{16}$. Neste sentido, modelos com efeitos aleatórios 
capturando a correlação entre observações deveriam ser utilizados. No entanto, análises preliminares mostraram que esses efeitos aleatórios não foram estatisticamente significativos e, desta forma, utilizamos o modelo multinomial padrão ${ }^{17}$.

O modelo multinomial utilizado é definido da seguinte forma :

$\ln \left(\frac{\pi_{\mathrm{ri}}}{\pi_{3 \mathrm{i}}}\right)=\beta^{\prime} \mathrm{x}_{\mathrm{i}} \quad \mathrm{r}=1,2$

onde $\pi_{\mathrm{ri}}$ é a probabilidade da i-ésima mulher ser classificada na categoria $r$ (necessidade para espaçar, $r=1$, e necessidade para limitar, $r=2$ ) e $\pi_{3 \mathrm{i}}$ é a probabilidade de ser classificada na categoria de referência 3 , que é composta por mulheres sem necessidade por anticoncepção. $\beta$ é o vetor associado à matriz de variáveis $\mathrm{x}$, que pode conter variáveis individuais e variáveis da área de residência.

Neste estudo utilizou-se a seguinte estratégia de modelagem. Primeiro, todas as variáveis foram incluídas. Aquelas estatisticamente significativas no nível de $5 \%$, por meio do teste da razão de verossimilhança, foram selecionadas. A seguir todas as interações de segunda ordem foram avaliadas. As análises foram implementadas utilizando-se o software SAS ${ }^{18}$.
Foi utilizado um conjunto de variáveis referentes às mulheres entrevistadas: região (Norte, Nordeste, Centro-Oeste, Sudeste e Sul), situação de residência (Rural e urbano), idade, número de filhos, educação (03, 4-8 e 9+), religião (católica praticante, católica não-praticante e não católica), raça/ cor (branca, parda e preta), mulher que trabalha (sim e não), acesso à mídia (assiste ou não TV pelo menos uma vez por semana). Duas variáveis contextuais no âmbito do município onde as mulheres residem foram incluídas na análise: Índice de Desenvolvimento Humano (IDH) e número de médicos por mil habitantes.

\section{Resultados}

A metodologia proposta por Westoff e Ochoa $^{7}$ está ilustrada no Esquema 1. Primeiramente foram selecionadas as mulheres unidas de 15 a 49 anos de idade (7584) que, por definição, estariam expostas ao risco da concepção. Entre as mulheres unidas foram eliminadas aquelas que faziam uso de anticoncepção seja para espaçar $(14,0 \%)$ ou para limitar os nascimentos $(62,8 \%)$.

As não usuárias de métodos anticoncepcionais $(23,3 \%)$ foram divididas em dois grupos: grávidas ou amenorréicas $(7,4 \%)$ e

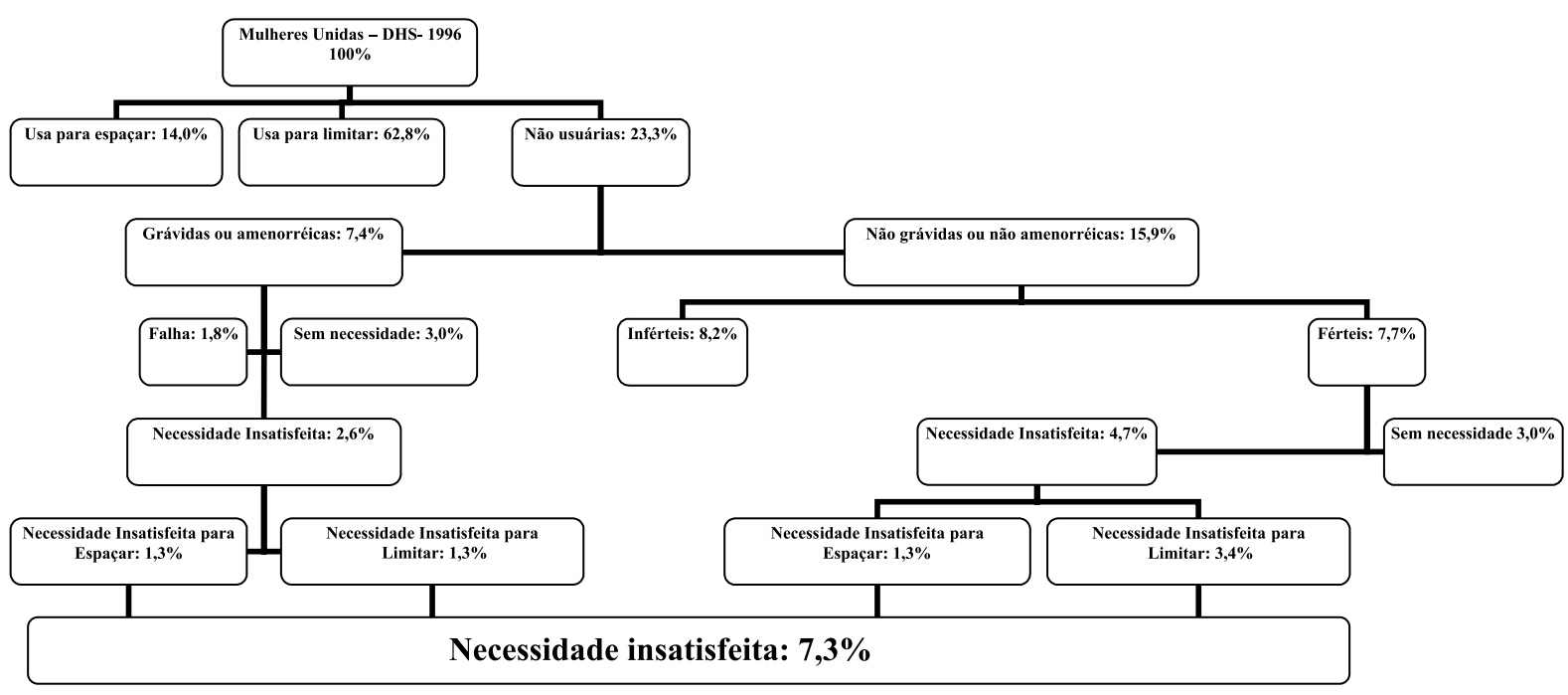

Esquema 1 - Necessidade insatisfeita de métodos anticoncepcionais, Brasil, PNDS-1996 Chart 1 - Unmet need for contraceptive methods, Brazil, PNDS, 1996 
Tabela 1 - Distribuição proporcional da necessidade insatisfeita por métodos anticoncepcionais, segundo as regiões do Brasil, PNDS-1996.

Table 1 - Proportion of unmet need for contraceptive methods, according to regions of Brazil, PNDS, 1996.

\begin{tabular}{lccccc}
\hline $\begin{array}{l}\text { Necessidade } \\
\text { Insatisfeita }\end{array}$ & Norte & Nordeste & $\begin{array}{c}\text { Regiões } \\
\text { Centro-Oeste }\end{array}$ & Sudeste & Sul \\
\hline Espaçar & 3,6 & 4,7 & 2,4 & 1,8 & 1,3 \\
Limitar & 5,7 & 8,2 & 2,4 & 3,8 & 2,6 \\
Total & 9,3 & 12,9 & 4,8 & 5,6 & 3,9 \\
\hline
\end{tabular}

não grávidas e não amenorréicas (15,9\%).

As mulheres grávidas ou amenorréicas cuja gravidez resultou de uma falha foram excluídas (1,8\%). Aquelas que declararam o desejo de sua última gravidez $(3,0 \%)$ foram classificadas como não tendo necessidade insatisfeita por métodos contraceptivos. As que desejaram a gravidez, mas declararam que esta ocorreu antes do previsto foram classificadas como tendo necessidade insatisfeita para espaçar $(1,3 \%)$ e as que não desejavam mais filhos foram classificadas como tendo necessidade insatisfeita para limitar (1,3\%).

As não grávidas e não amenorréicas foram classificadas em férteis $(7,7 \%)$ e inférteis $(8,2 \%)$. As mulheres inférteis são aquelas que, apesar de união contínua e sem uso de método contraceptivo, não tiveram filhos durante os últimos cinco anos. Também foram consideradas inférteis mulheres que declararam estar em amenorréia há mais de seis meses do início da pesquisa, mulheres na menopausa e aquelas com amenorréia primária, ou seja, que nunca menstruaram. As mulheres férteis que desejavam um filho adicional no momento da entrevista foram classificadas como não tendo necessidade insatisfeita por anticoncepção (3,0\%). As que desejavam um filho adicional, mas preferiam esperar pelo menos dois anos para tê-lo, foram classificadas como tendo necessidade insatisfeita para espaçar (1,3\%). Finalmente, aquelas que no momento da entrevista declararam não desejar algum filho adicional foram classificadas como tendo necessidade insatisfeita para limitar $(3,4 \%)$.

Ao final desse processo, 7,3\% das mu- lheres foram classificadas como tendo necessidade insatisfeita por anticoncepção, totalizando 1.105 observações, sendo 2,6\% para espaçar e $4,7 \%$ para limitar.

Na Tabela 1 encontra-se a distribuição proporcional de mulheres com necessidade insatisfeita segundo as regiões do país. A proporção de mulheres com necessidade insatisfeita, seja para espaçar ou limitar, é maior nas regiões mais pobres do país, isto é Nordeste e Norte.

Os resultados do modelo multinomial utilizado para identificar os fatores associados à necessidade insatisfeita por métodos anticoncepcionais estão apresentados na Tabela 2. Seis variáveis foram excluídas do modelo, cinco individuais e uma contextual, pois não foram estatisticamen-

Tabela 2 - Coeficientes das variáveis selecionadas pelo modelo multinomial, Brasil, PNDS-1996

Table 2 - Coefficients of the variables selected by the multinomial model, Brazil, PNDS, 1996

\begin{tabular}{lcc}
\hline Característica & Espaçar & Limitar \\
\hline Região (Ref: Nordeste) & & \\
Norte & $-0,596^{*}$ & $0,762^{*}$ \\
Centro-Oeste & 0,043 & $-0,477$ \\
Sudeste & $-0,421$ & $-0,341$ \\
Sul & $-0,813^{*}$ & $-0,845^{*}$ \\
Idade & $-0,122^{*}$ & $0,039^{*}$ \\
Número de filhos & 0,35 & $-0,508$ \\
Educação (Ref: 0-3) & & \\
4-8 & $-0,139$ & $-0,410^{*}$ \\
9+ & 0,010 & $-0,482^{*}$ \\
IDH & 1,031 & $-0,124$ \\
Interação & & \\
Número de filhos*IDH & $-0,037$ & $1,522^{*}$ \\
\hline * p<0,05 & &
\end{tabular}




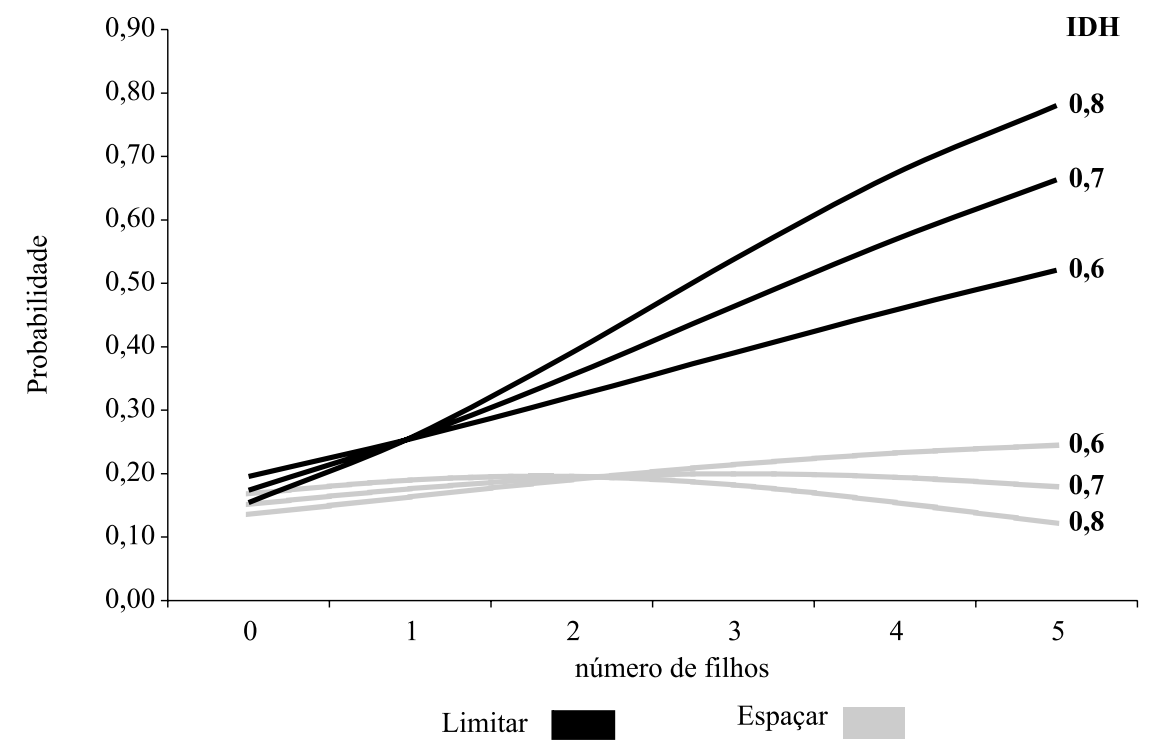

Gráfico 1 - Probabilidade de se ter necessidade insatisfeita por métodos anticoncepcionais, segundo número de filhos e IDH, Brasil, PNDS-1996

Graph 1 - Probability of having an unmet need for contraceptive methods according to number of children and HDI, Brazil, PNDS, 1996

Tabela 3 - Probabilidade de se ter necessidade insatisfeita para espaçar e limitar, Brasil, PNDS-1996

Table 3 - Probability of having unmet need for spacing and limiting, Brazil, PNDS-1996

\begin{tabular}{lcc}
\hline Característica & Espaçar & Limitar \\
\hline Região & & \\
Norte & 0,175 & 0,281 \\
Nordeste & 0,217 & 0,412 \\
Centro-Oeste & 0,266 & 0,299 \\
Sudeste & 0,177 & 0,363 \\
Sul & 0,150 & 0,274 \\
Idade & & \\
15 & 0,602 & 0,134 \\
20 & 0,434 & 0,217 \\
25 & 0,277 & 0,310 \\
30 & 0,161 & 0,401 \\
35 & 0,086 & 0,481 \\
40 & 0,044 & 0,549 \\
45 & 0,022 & 0,608 \\
Educação & & \\
$0-3$ & 0,191 & 0,423 \\
$4-8$ & 0,200 & 0,338 \\
$9+$ & 0,230 & 0,311 \\
\hline
\end{tabular}

te significativas ao nível de 5\%: situação de residência, raça, religião, acesso à mídia, mulher que trabalha e número de médi- cos por mil habitantes. O modelo apresentou uma interação, estatisticamente significativa, entre o número de filhos tidos e o índice de desenvolvimento humano (IDH).

Para melhor interpretar os resultados do modelo multinomial, as probabilidades de ter uma necessidade insatisfeita foram calculadas segundo categorias das variáveis selecionadas, tomando as demais variáveis no seu valor médio ${ }^{17}$. As probabilidades referentes aos efeitos principais das variáveis e da interação entre número de filhos e o IDH do município encontram-se respectivamente descritas na Tabela 2 e no Gráfico 1.

Corroborando os achados da análise descritiva, o resultado do modelo revela o Nordeste como sendo a região com maior probabilidade de encontrarmos mulheres unidas com necessidade insatisfeita, sendo que prevalece a necessidade insatisfeita para limitar. Na realidade, a necessidade insatisfeita para limitar é maior em todas as regiões do país. As regiões Norte e Sul apresentam as menores probabilidades de necessidade insatisfeita. Cabe ressaltar que, na região Norte, apenas a área urbana foi amostrada, o que pode explicar parcialmente este resultado. 
A idade da mulher foi incluída no modelo como uma variável contínua e apresenta uma forte associação com a necessidade insatisfeita. Conforme esperado, na medida em que as mulheres envelhecem aumenta a necessidade para limitar e decresce a necessidade para espaçar. O nível de escolaridade das mulheres mostra-se inversamente associado à necessidade insatisfeita para limitar. Quanto maior a escolaridade das mulheres, menor a necessidade insatisfeita para limitar.

As probabilidades de uma mulher ter necessidade insatisfeita por métodos anticoncepcionais, para limitar ou espaçar, segundo números de filhos e nível o IDH do município, são apresentadas no Gráfico 1. Três valores foram selecionados para o IDH - 0,6, 0,7 e 0,8 -correspondendo aproximadamente ao primeiro quartil, mediana e terceiro quartil, respectivamente. Nos municípios com IDH baixo, a probabilidade de uma mulher ter necessidade insatisfeita por métodos anticoncepcionais para espaçar mostra uma associação direta com o número de filhos, enquanto nos municípios com IDH alto, a relação é inversa. Nenhuma relação é observada nos municípios com IDH médio $(0,7)$. No que se refere à necessidade insatisfeita para limitar, a relação com o número de filhos é sempre direta, independentemente do nível do IDH. No entanto, vale ressaltar que a inclinação é maior nos municípios com IDH mais elevado. A título de ilustração, a probabilidade de uma mulher com cinco filhos ter uma necessidade para limitar é aproximadamente 50\% maior nos municípios com IDH alto $(0,8)$, quando comparados com os municípios com IDH baixo $(0,6)$.

\section{Discussão}

No Brasil, a prevalência do uso de contraceptivo é elevada e a taxa de fecundidade total está quase no nível de reposição. No entanto, observa-se que 7,3\% de mulheres casadas possuem necessidade insatisfeita por contracepção, sendo $4,7 \%$ para limitar e $2,6 \%$ para espaçar.
Ainda que este valor seja baixo, quando comparado com estimativas realizadas para a América Latina em $1996(17 \%)^{19}$, ele não é desprezível, pois representa mais de três milhões de mulheres em idade reprodutiva. São mulheres que querem adiar ou limitar os nascimentos, mas que por alguma razão não estão usando qualquer tipo de método contraceptivo. Desta forma, ficam expostas ao risco de uma gravidez indesejada, podendo assim recorrer ao aborto clandestino. São as mais pobres, no entanto, que vão tê-lo em condições inseguras.

Note que a necessidade insatisfeita para limitar é bastante elevada nos estratos sociais mais vulneráveis da população. São justamente as mulheres com menor escolaridade e residentes na região Nordeste que apresentam maior necessidade para limitar.

A interação entre a variável individual número de filhos e a contextual nível de desenvolvimento humano ressalta a diferença na percepção das mulheres quanto à necessidade para limitar, segundo o nível do IDH. Por exemplo, mulheres com cinco filhos residentes em municípios com IDH elevado têm probabilidade $67 \%$ maior de ter necessidade insatisfeita por métodos anticoncepcionais do que mulheres com cinco filhos residentes em municípios com IDH baixo. As motivações que podem estar relacionadas à percepção das mulheres de limitar nascimento nos municípios com IDH elevado, podem advir de um padrão reprodutivo diferenciado decorrente de estilos e perspectivas de vida diferentes, na medida em que estas mulheres teriam maior chance de realização em outras áreas, e não somente através da maternidade. As oportunidades são maiores em municípios com maior nível educacional, que é um dos componentes do cálculo do IDH. Cabe destacar o efeito de um outro componente, a mortalidade. Alguns estudos têm destacado a queda da mortalidade como um elemento importante para o declínio da fecundidade ${ }^{20,21}$. É provável que em municípios com mortali- 
dade elevada, as mulheres sejam menos propensas a limitar a prole.

Uma limitação desse estudo diz respeito à população sob investigação, na medida em que a necessidade insatisfeita foi estimada apenas para mulheres casadas. Dixon \& Germain ${ }^{19}$ ressaltam a importância de se calcular a necessidade insatisfeita entre as mulheres não unidas, tendo em vista que uma proporção significativa dessas mulheres é sexualmente ativa. Apesar da PNDS-96 ter coletado informações sobre atividade sexual das entrevistadas e a BEMFAM apresentar em seu relatório estimativas da necessidade insatisfeita por anticoncepção para esse grupo de mulheres (2\%), é difícil inferir se essas mulheres estão de fato expostas a gravidez indesejada. Além disso, não existe um critério claro para identificar, nesse grupo, as mulheres fecundas. Esse é um campo que certamente deve ser explorado, principalmente em estudos comparativos com ênfase na informação sobre a atividade sexual das mulheres não unidas.

\section{Considerações finais}

O conhecimento das características das mulheres unidas com necessidade insatisfeita por contracepção pode facilitar a qualificação dos programas de planejamento familiar através da identificação das principais barreiras no uso de métodos anticoncepcionais para a população em idade reprodutiva que deseja limitar ou espaçar o nascimento dos filhos.

A insatisfação da usuária e o acesso restrito aos diferentes métodos contraceptivos, podem resultar em elevada taxa de descontinuação de métodos anticoncepcionais no Brasil (43\% das usuárias descontinuam nos primeiros 12 meses $)^{6}$, contribuindo assim para o aumento no número de mulheres com necessidade insatisfeita por métodos anticoncepcionais, principalmente quando a descontinuação não resulta em uma troca imediata de métodos. Assim, há necessidade de se avaliar a adequabilidade dos métodos contraceptivos, pois estar usando um método não necessariamente significa estar satisfeita com o mesmo.

O presente estudo evidencia a vulnerabilidade das mulheres mais pobres que devem se constituir na população-alvo dos programas de atenção à saúde da mulher.

As práticas de planejamento familiar devem garantir às mulheres um ambiente humanizado que facilite a reflexão sobre as preferências reprodutivas, com disponibilidade de informações e acesso facilitado aos diversos métodos contraceptivos. Nesse contexto, as políticas públicas voltadas para o planejamento familiar devem ter como meta os princípios estabelecidos na Conferência do Cairo de 1994, com vista a ajudar os casais e os indivíduos a satisfazer os seus objetivos reprodutivos, evitando assim a gravidez não desejada.

\section{Referências}

1. IBGE - Censo Demográfico 2000 Nupcialidade e fecundidade. Disponível em: URL: http:// www.ibge.gov.br Acessado em 15 de novembro de 2004.

2. Martine G. Brazil's fertility decline, 1965-95: a fresh look at key factors. Population and Development Review 1996; 22: 47-75.

3. Perpetuo IHO. Contracepção e declínio da fecundidade na Região Nordeste. Rev Bras Estud Popul 1998; 15(1): 4356.
4. Perpétuo IHO, Aguirre MAC. O papel da esterilização feminina nos diferenciais sócio-econômicos do declínio da fecundidade no Brasil. In: Anais do $11^{\circ}$ Encontro Nacional de Estudos Populacionais; 1998; Caxambu (Br). Minas Gerais: ABEP; 1998. v. 1. p. 2997-3024.

5. Alan Guttmacher Institute(US). Aborto clandestino: una realidade latinoamericana. New York: The Institute; 1994. Disponível em: URL http:/ /www.guttmacher.org/pubs/ journals/2300497.htl Acessado em 23 de fevereiro de 2005. 
6. Badiani R, Ferreira I, Ochoa L, Patarra N, Womg L, Camarano A, et al. Brasil: pesquisa nacional sobre demografia e saúde 1996. Rio de Janeiro: BENFAM-DHS; 1997.

7. Westoff CF, Ochoa LH. Unmet need and the demand for family planning. In: Demographic and Health Surveys Comparatives Studies. No. 5. Columbia (USA) Maryland: Institute for Resource Development/Macro Systems, Inc.; 1991.

8. Robey B, Ross J, and Bhushan I.. Meeting unmet need: New strategies. Population Reports 1996 ; 43: 1-35.

9. Costa AM. Atenção Integral à Saúde das Mulheres: QUO VADIS? Uma Avaliação da Integralidade na Atenção à Saúde das Mulheres no Brasil [tese de doutorado]. Brasília: Faculdade de Ciências da Saúde da Universidade de Brasília; 2004.

10. Ashford L. Unmet need for family planning: Recent trends and their implications for programs. PRB. Unmet Need for Family Planning 2003; 1-8. Disponível em: URL: http://www.phishare.org/documents/PRB/214/email Acessado em 23 de agosto de 2005.

11. Casterline JB, Sinding S. Unmet need for family planning in developing countries and implications for population policy. Population and Development Review 2000; 26(4): 691-723.

12. Westoff CF. The potential demand for family planning: a new measure of unmet need and estimates for five Latin American countries. International Family Planning Perspectives 1988; 14(2): 45-53.

13. Westoff CF, Pebley AR. Alternative measures of unmet need for family planning in developing countries. International Family Planning Perspectives 1981; 7(4): 126-36.
14. Calle M. Estudo dos componentes da dinâmica reprodutiva na Bolívia [tese de doutorado]. Minas Gerais: Faculdade de Ciência Econômicas da Universidade Federal de Minas Gerais; 2003.

15. Nortman DL. Measuring the unmet need for contraception to space and limit births. International Family Planning Perspectives 1982; 8(4): 125-34.

16. Goldstein H. Multilevel statistical models. London: Edward Arnold; 1995.

17. Retherford RD, Choe MK. Statistical models for causal analysis. New York: Wiley; 1993.

18. SAS Institute. In SAS/STAT User's Guide. Version 6. Cary (North Carolina): SAS Institute Inc; 1993.

19. Dixon MR., Germain A. Stalking the elusive unmet need for family planning. Studies in Family Planning 1992; 23(5): 330-5.

20. Lindstrom, DP, Betemariam B. "The impact of war, famine, and economic decline on marital fertility in Ethiopia," Demography 1999;36(2): 247-26. (CHECAR PÁGS. ARTIGO)

21. Agadjanian, Victor and Ndola Prata. "War, peace, and fertility in Angola” Demography 2002; 39(2): 215-231.

Recebido em: 12/06/06

Versão final reapresentada em: 31/03/07

Aprovado em: 10/04/07 